\title{
太子参环肽B(Heterophyllin B, HB)的酶环化反应
}

\author{
贾爱群 ${ }^{\oplus 0}$ 李 湘 ${ }^{\circledR}$ 谭宁华 ${ }^{\circledR}$ 刘小烛 ${ }^{\circledR}$ 沈月毛 ${ }^{\circledR}$ 周 俊 ${ }^{* *}$
}

(1) 中国科学院昆明植物研究所 植物化学与西部植物资源持续利用国家重点实验室, 昆明 650204; (2) 中国科学院 研究生院，北京 100039; (3) 杭州中肽有限公司，杭州 310018; (4) 西南林学院，昆明 650204)

\begin{abstract}
摘要 利用从Pseudostellaria heterophylla提取分离的粗酶 PH-1, 以链八肽 $\mathrm{NH}_{2}-\mathrm{Gly}^{1}$-Gly ${ }^{2}$-LeuPro-Pro-Pro-Ile-Phe-COOH (4)为底物成功地进行了太子参环肽B(heterophyllin B (HB)) 的环化反应, 并利用TLC, HPLC, MS, NMR以及同位素标记等手段对反应产物进行了分析和确证.
\end{abstract}

\section{关键词 太子参 环肽 太子参环肽 B 酶环化 同位素标记}

环肽(cyclopeptides, cyclic peptides)是一类重要 的天然产物，按照其来源的不同可分为植物环肽、动 物环肽和微生物环肽. 在过去的十多年中, 我们课题 组从植物里共分离鉴定了约 100 个环肽 ${ }^{[1]}$. 随着环肽 从自然界的不断发现, 其独特新颖的结构引起了诸 多学科的关注, 最近已经有关于微生物环肽的报道, 如 tyrocidine $\mathrm{A}^{[2 \sim 4]}$. 但植物环肽, 尤其是高等植物环 肽的生物合成途径一直困扰着我们.

太子参是石竹科植物异叶假繁缕 (PseudosteIlaria heterophylla (Mig.) Pax ex Pax et Hoffm)的块茎, 是一种著名的传统中药 ${ }^{[5]}$. 因为太子参环肽 $\mathrm{B}$ 较其它植 物环肽在植物体中含量大、结构明确, 组成氨基酸全 为 $I$ 型, 且原植物易得 ${ }^{[6]}$, 因此本文以太子参为研究对 象, 以太子参环肽 $\mathrm{B}$ (heterophyllin $\mathrm{B}, \mathrm{HB}$ )为目标产物 (TC)(结构如式 1)进行了植物环肽酶环化的初步探讨.

关于 HB 的酶环化反应, 我们遵循从简单入手的 原则( $a b$ initio), 并结合微生物环肽的合成途径, 认为

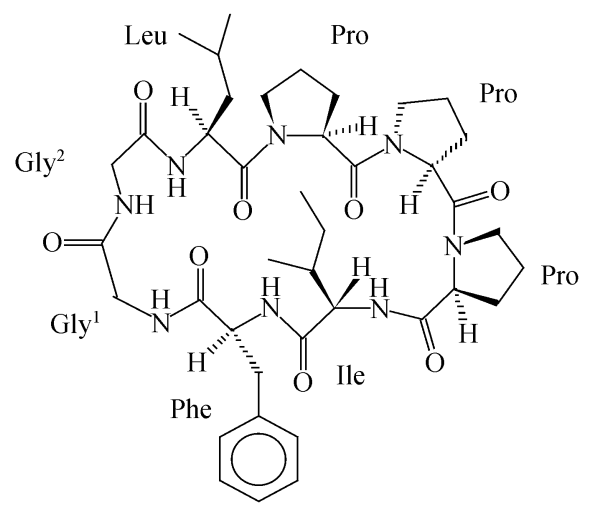

式 1 太子参环肽 B (HB)

植物环肽有可能是在某一或某些环化酶的作用下， 利用体内已有的链肽一步形成相应的植物环肽, 即:

链 $n$ 肽 $\stackrel{\text { 环化酶 }}{\longrightarrow}$ 环 $n$ 肽

在此假说下, 要合成 HB, 理论上需要合成 8 个 链八肽, 但由于环肽中存在 3 个位阻很大的脯氨酸 
(Pro), 因此在与 3 个脯氨酸相连接的 4 个位点形成环 肽的可能性很小, 故利用肽的固相合成方法(SPPS)只 需要合成 4 个链八肽作为底物即可. 即:

1: $\mathrm{NH}_{2}$-Phe-Gly ${ }^{1}$-Gly ${ }^{2}$-Leu-Pro-Pro-Pro-Ile-COOH

2: $\mathrm{NH}_{2}$-Gly ${ }^{2}$-Leu-Pro-Pro-Pro-Ile-Phe-Gly ${ }^{1}-\mathrm{COOH}$

3: $\mathrm{NH}_{2}$-Leu-Pro-Pro-Pro-Ile-Phe-Gly ${ }^{1}-\mathrm{Gly}^{2}-\mathrm{COOH}$

4: $\mathrm{NH}_{2}$-Gly ${ }^{1}$-Gly ${ }^{2}$-Leu-Pro-Pro-Pro-Ile-Phe-COOH

上述链八肽的所有氨基酸残基均是 $l$ 构型.

该环化反应中所需要的酶是经过下列分离步骤 得到的: (i) 快速洗去新鲜太子参上的泥土后, 利用 细胞粉碎的方法在 $4^{\circ} \mathrm{C}$ 下, 提取总蛋白; (ii) 利用 Sephadex G-50 和阳离子交换树脂 CM-52 进行分离纯 化, 得到粗环化酶 PH-1.

接着在下列反应条件下, 进行 HB 的环化反应:

(1) $\mathrm{NH}_{2}$-Phe-Gly ${ }^{1}$-Gly ${ }^{2}$-Leu-Pro-Pro-Pro-Ile-COOH

(2) $\mathrm{NH}_{2}$-Gly ${ }^{2}$-Leu-Pro-Pro-Pro-Ile-Phe-Gly ${ }^{1}-\mathrm{COOH}$

(3) $\mathrm{NH}_{2}$-Leu-Pro-Pro-Pro-Ile-Phe-Gly ${ }^{1}$-Gly ${ }^{2}-\mathrm{COOH}$

(4) $\mathrm{NH}_{2}$-Gly ${ }^{1}-\mathrm{Gly}^{2}$-Leu-Pro-Pro-Pro-Ile-Phe-COOH

$$
\stackrel{\mathrm{PH}-1}{\longrightarrow} \mathrm{HB}
$$

缓冲液: Tris: $\mathrm{pH}=8.0$; 温度: $24^{\circ} \mathrm{C}$; 时间: $3 \mathrm{~h}$; 底物 1 4：环化酶 =1：8; 反应(4)得率: $27.28 \%$.

在相同条件下, 分别以链八肽 1 4 为底物进行环 化反应，发现仅链八肽 4 在粗酶 PH-1 作用下呈阳性 反应, 而链八肽 1 3 则没发生反应. 为证明反应是否 呈阳性或阴性反应，在严格的相同条件下，反复进行 了底物 1 4 的平行反应, 进一步证明了仅底物 4 呈阳 性反应, 平均得率为 $27.28 \%$.

为了进一步验证反应 4 是否假阳性反应, 我们利 用SPPS方法对底物 4 上与苯丙氨酸相连的甘氨酸上 的羰基进行了 ${ }^{13} \mathrm{C}$ 同位素标记，即：

$\mathrm{NH}_{2}$-Gly ${ }^{1}{ }^{13} \mathrm{CO}-\mathrm{Gly}^{2}$-Leu-Pro-Pro-Pro-Ile-Phe-COOH.

在相同反应条件下，证明了反应 4 是阳性反应. 接着, 我们利用半制备 HPLC 技术分离纯化了反应产 物, 并利用诸多分析手段如 TLC, HPLC, MS 和 NMR 对反应液或反应产物进行了分析.

利用环肽的TLC检测方法 ${ }^{11]}$, 发现反应 4 的产物 呈现环肽的特征显色，说明在反应条件下，链八肽 4 进行了环化反应(图 1).

接着我们对反应 1 4 各反应液进行了 HPLC 分析 (图 2), 发现底物 1 4 的保留时间分别为 7.196, 12.533,

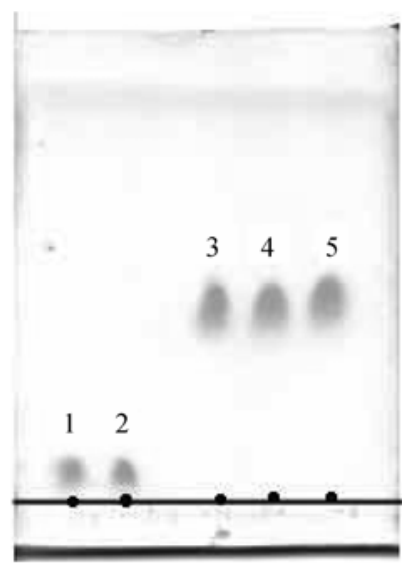

图 1 TLC 检测图

硅胶G板; 流动相: $\mathrm{CHCl}_{3}: \mathrm{CH}_{3} \mathrm{OH}=9: 1 ; 1,4 ; 2,{ }^{13} \mathrm{C}$ 标记 $4 ; 3,{ }^{13} \mathrm{C}$ 标 记HB(酶合成产物); 4, HB(酶合成产物); 5, HB(标准品)

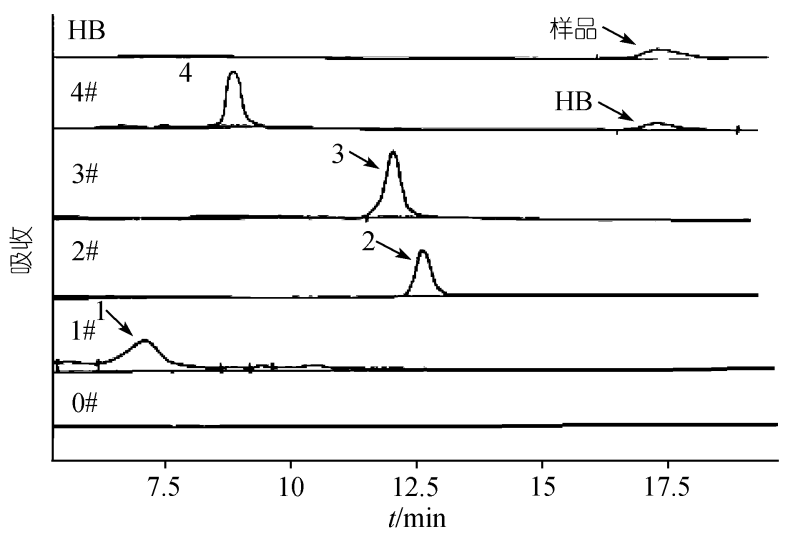

图 2 HPLC 检测图

仪器: Agilent 1100 (Agilent Technologies, Wilmington, USA); 层析柱: Accurasil C-18 (5 $\mu \mathrm{m}, 250 \mathrm{~mm} \times 4.6 \mathrm{~mm})$; 流动相: $70 \% \mathrm{H}_{2} \mathrm{O}$ 和 $30 \%$ $\mathrm{CH}_{3} \mathrm{CN}$; 检测波长: $192 \mathrm{~nm}$; 流速: $1 \mathrm{~mL} / \mathrm{min}$; $4 \#$ (3\#, 2\#, 1\#): 粗酶 +4

$$
(3,2,1) \text {; 0\#: 酶 }
$$

11.904 和 $8.831 \mathrm{~min}$, 并发现反应 $\mathbf{4}$ 有产物峰出现, 其 保留时间 $17.318 \mathrm{~min}$, 通过与对照品 HB 的保留时间 (17.340 min) 比较, 证明反应 4 的产物有可能是 HB.

对纯化的反应产物进行了 $\mathrm{FAB}^{+}-\mathrm{MS}$ 分析, 分别 得到 2 个分子离子峰: $779\left([\mathrm{M}+1]^{+}\right)$和 $780\left([\mathrm{M}+1]^{+}\right)$, 可 能是反应产物 $\mathrm{HB}$ 和 ${ }^{13} \mathrm{C}$ 标记HB的分子离子峰(图 3).

为了进一步证明环化反应的成功与否, 我们利 用 $\mathrm{FAB}^{+}-\mathrm{MS}$ 对反应产物 ${ }^{13} \mathrm{C}$ 标记HB进行了同位素峰 组分析, 发现产物中的同位素组成为: $\left[{ }^{13} \mathrm{C} \mathrm{HB}\right] / \mathrm{HB}$ 


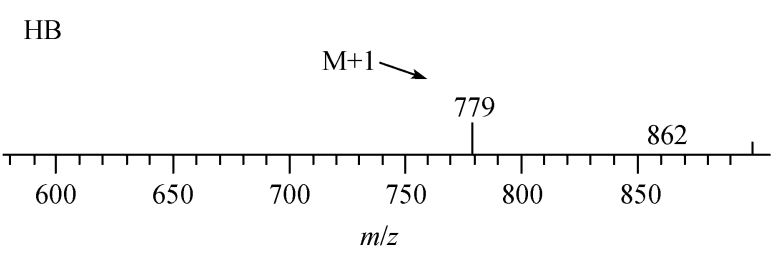

${ }^{13} \mathrm{C}$ 标记HB

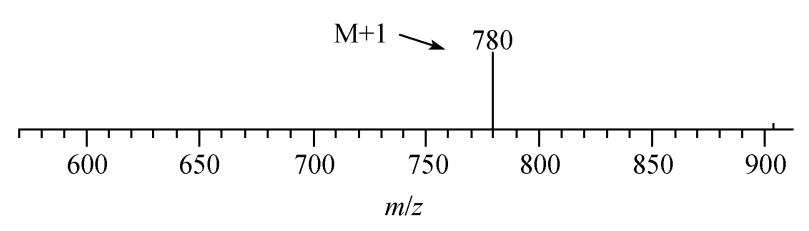

图 $3 \mathrm{HB}$ 和 ${ }^{13} \mathrm{C}$ 标记 $\mathrm{HB}$ 的 $\mathrm{FAB}^{+}-\mathrm{MS}$ 谱

$=8:$. 通过反应产物 ${ }^{13} \mathrm{C}$ 标记 $\mathrm{HB}$ 和反应底物 ${ }^{13} \mathrm{C} \mathbf{4}$ 比较发现：前者的 ${ }^{13} \mathrm{C}$ 丰度为 $89.13 \%$, 后者的为 $92.46 \%$, 即在反应过程中发生了部分 ${ }^{13} \mathrm{C}$ 衰变(表 1$)$.

表 $1{ }^{13} \mathrm{C}$ 标记 4 和 ${ }^{13} \mathrm{C}$ 标记HB的FAB ${ }^{+}-\mathrm{MS}$ 丰度

\begin{tabular}{cccccc}
\hline & $\mathrm{M}$ & $\mathrm{M}+1$ & $\mathrm{M}+2$ & $\mathrm{M}+3$ & $\mathrm{M}+4$ \\
\hline${ }^{13} \mathrm{C} \mathbf{4}$ & 8.16 & 100.00 & 83.33 & 37.38 & 13.24 \\
& $(797)$ & $(798)$ & $(799)$ & $(800)$ & $(801)$ \\
${ }^{13} \mathrm{C} \mathrm{HB}$ & 12.19 & 100.00 & 83.11 & 14.36 & 7.13 \\
& $(779)$ & $(780)$ & $(781)$ & $(782)$ & $(783)$ \\
\hline
\end{tabular}

最后我们对反应产物 ${ }^{13} \mathrm{C}$ 标记 $\mathrm{HB}$ 和 $\mathrm{HB}$ 进行了 ${ }^{13} \mathrm{C}$ NMR分析并与文献对照知, 171.820 是 $\mathrm{HB}$ 中 $\mathrm{Gly}^{1}$ 的 羰基峰, 而 171.913 是 ${ }^{13} \mathrm{C}$ 标记HB中 $\mathrm{Gly}^{1}$ 的羰基峰, 较 HB中的Gly ${ }^{1}$ 羰基峰化学位移向低场位移 0.093, 而底 物 4 中 Gly ${ }^{1}$ 的羰基峰为 169.846, 与 171.913 相比, 化 学位移相差 2.067, 显然 171.913 应该是反应产物 ${ }^{13} \mathrm{C}$ 标记HB中 Gly ${ }^{1}$ 的羰基峰, 进一步证明反应 4 是一阳 性反应, 即在反应条件下, 成功地合成了目标产物 $\mathrm{HB}$ (图 4).

上述多种分析手段以及同位素标记方法的综合 运用证明底物 4 在反应条件下可以成功地合成目标 产物 HB.

在进行该反应之前, 我们对植物环肽尤其石竹 科环肽中的氨基酸残基和二肽残基进行了数学统计, 发现甘氨酸和苯丙氨酸残基在出现几率很大, 并且 二者形成的二肽残基在环肽中出现的几率也较大,
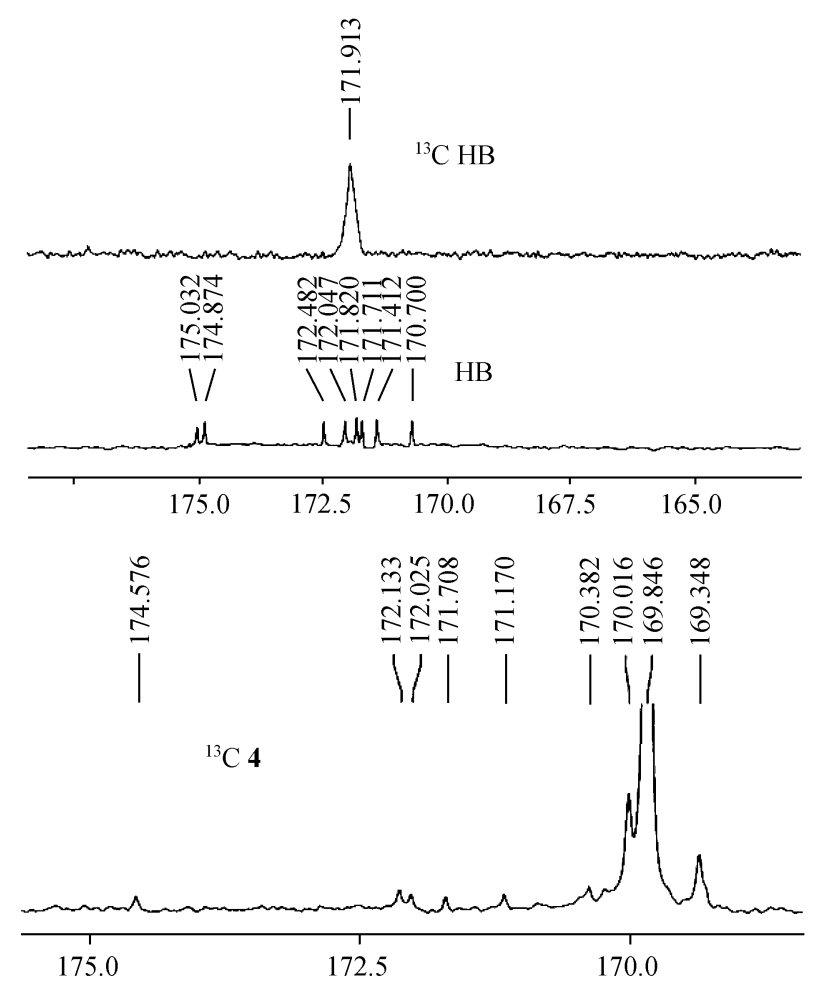

\section{图 $4{ }^{13} \mathrm{C}$ 标记 4, HB 和 ${ }^{13} \mathrm{C}$ 标记HB的 ${ }^{13} \mathrm{C}$ NMR谱 氝代溶剂: $\mathrm{C}_{5} \mathrm{D}_{5} \mathrm{~N}, 125 \mathrm{MHz}$}

因此我们预测, 甘氨酸和苯丙氨酸可能是由链肽形 成环肽的反应位点，该反应的成功也证明了我们的 预测.

在本文中我们只是进行了 $\mathrm{HB}$ 的酶环化反应研 究, 至于由甘氨酸和苯丙氨酸形成的二肽残基是否 是该类环肽环化的位点还需进一步证明.

另外我们只是对植物环肽进行了初步的酶环化 反应, 其中所用的环化酶PH-1 是一粗酶, 还有待进 一步分离纯化, 是单一酶还是多酶共同作用尚不得 而知, 根据微生物环肽的环化反应推测 ${ }^{[2 \sim 4]}$, 植物环 肽也可能是多酶作用的结果. 通过对粗酶 PH-1 的分 离纯化也初步证明环肽HB的环化是多酶作用.

该研究是植物环肽环化反应的初步探讨, 目前 还没发现关于植物环肽的环化反应文献报道. 为数不 多的几篇文献则集中在微生物环肽的环化研究上 ${ }^{[2,3]}$.

致谢感谢中国科学院昆明植物研究所植物化学与 西部植物资源国家重点实验室分析测试中心的分析 
测试, 感谢中国科学院昆明植物研究所植物化学与 西部植物资源国家重点实验室胡忠研究员的有益帮 助.

\section{参考 文 献}

1 Zhou J, Tan N H. Application of a new TLC chemical method for detection of cyclopeptides in plants. Chinese Science Bulletin 2000, 45(20): 1825 1831

2 Kohli R M, Walsh C T, Burkart M D. Biomimetics synthesis and optimization of cyclic peptide antibiotics. Nature, 2002, 418(6898): 658 661[DOI]
3 Kohli R M, Burke M D, Tao J H, Walsh C T. Chemoenzymatic route to macrocyclic hybrid peptide/polyketide-like molecules. J Am Chem Soc, 2003, 125(24): 7160 7161[DOI]

4 Jackson D Y, Burnier J P, Wells J A. Enzymatic cyclization of linear peptides esters using subtiligase. J Am Chem Soc, 1995, 117(2): 819 820[DOI]

5 中国医学科学院. 中药志, 第 2 卷. 北京: 人民卫生出版社, 1995. 266 268

6 Tan N H, Zhou J, Chen C X, Zhao S X. Cyclopeptides from the root of Pseudostellaria heterophylla. Phytochemistry, 1993, 32(5): 1327 1330[DOI] 